Case Report

\title{
Isolated Left Main Coronary Artery Stenosis after Thoracic Radiation Therapy: To Operate or Not to Operate
}

\author{
Osama Alsara, ${ }^{1}$ Ahmad Alsarah, ${ }^{1}$ Jagadeesh K. Kalavakunta, ${ }^{2}$ \\ Heather Laird-Fick, ${ }^{1}$ and George S. Abela ${ }^{2}$ \\ ${ }^{1}$ Michigan State University, Department of Internal Medicine, B-301 Clinical Center, East Lansing, MI 48824, USA \\ ${ }^{2}$ Michigan State University, Division of Cardiovascular Disease, B-208 Clinical Center, East Lansing, MI 48824, USA \\ Correspondence should be addressed to Osama Alsara; osama.alsara@hc.msu.edu
}

Received 20 June 2013; Accepted 6 November 2013

Academic Editor: Bruno Megarbane

Copyright (C) 2013 Osama Alsara et al. This is an open access article distributed under the Creative Commons Attribution License, which permits unrestricted use, distribution, and reproduction in any medium, provided the original work is properly cited.

Radiation therapy of neoplasms involving the chest or mediastinum results in a wide spectrum of cardiac complications including coronary artery disease, which can present in patients with few or no traditional cardiac risk factors. We report a case of radiation induced coronary artery disease in a 60-year-old female with a history of stage IIIA nonsmall cell lung carcinoma which was diagnosed eight years earlier and treated with chemotherapy and radiotherapy. She presented to the hospital with atypical chest pain that had occurred intermittently over the preceding week. Her initial electrocardiogram and cardiac enzymes were within normal limits. However, following an indeterminate exercise nuclear stress test, she developed chest pain and elevated cardiac enzymes. Coronary angiography demonstrated $90 \%$ stenosis of the left main coronary artery ostium, without any evidence of atherosclerotic disease or stenosis in other coronary arteries. She underwent surgical revascularization, which revealed dense adhesions surrounding the heart. During surgery, she developed severe bleeding and died. Coronary artery disease can present within years of radiation exposure, and ostial lesions are typical. Treatment is often challenging because of the effects of radiation on other tissues and the risks of revascularization procedures. Therefore, a multidisciplinary team approach should be considered.

\section{Introduction}

For the past few decades, radiation has been used effectively in the treatment of neoplasms involving the chest and mediastinum, including breast cancer, lymphomas, and lung cancer. However, radiotherapy of the chest can have a number of cardiac complications including pericarditis, pericardial effusions, conduction disorders, pancarditis, functional valvular defects, and coronary artery disease (CAD) [1]. CAD has been reported more commonly with Hodgkin's lymphoma, presumably because these patients tend to be younger and survive longer [2]. In this report we describe a 60-yearold woman with isolated left main coronary ostial stenosis diagnosed eight years after chest irradiation performed for stage IIIA nonsmall cell lung cancer. This case emphasizes the importance of clinical suspicion, prognostication, and the balancing risks and benefits of competing therapies.

\section{Case Presentation}

A 60-year-old woman with a history of lung cancer currently in remission was admitted with complaints of chest pain. She had recurrent substernal chest pain, increasing in severity, without radiation or associated symptoms during the preceding week. She denied any specific alleviating or provoking factors.

Past medical history was significant for hypertension and stage IIIA nonsmall cell lung carcinoma which was diagnosed eight years earlier. She had been treated with chemotherapy and radiotherapy. One year later she developed a malignant pericardial effusion, treated with a pericardial window, and recurrent pleural effusions, which were drained with pleural catheters. During the next six years, she was in full remission as confirmed by annual chest computed tomography (CT). Her medications included aspirin, metoprolol, iron, vitamin 


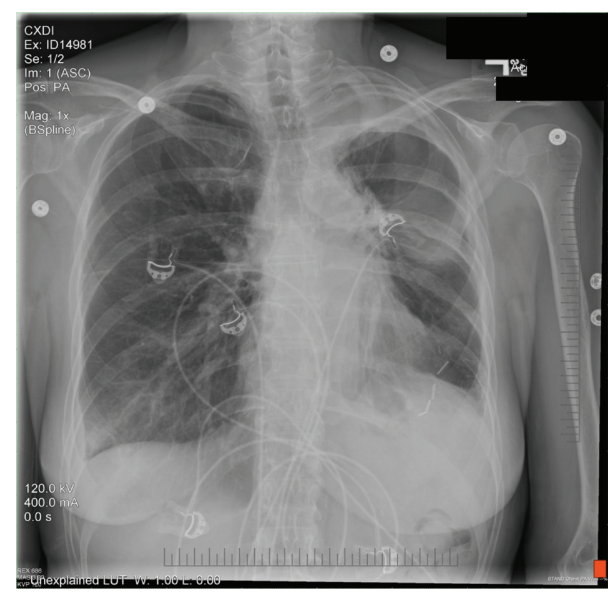

FIgUre 1: Posterior to anterior chest X-ray shows left perihilar parenchymal scars, surgical clips, and postoperative changes in the chest.

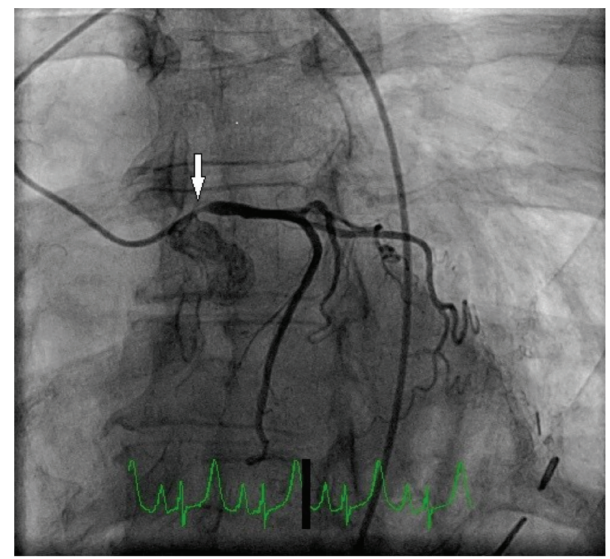

FIgURE 2: Coronary angiography with a right anterior oblique (RAO)/caudal projection. Arrow points to severe isolated ostial stenosis of left main coronary artery.

B12, and gefitinib. She was a nonsmoker and drinks alcohol occasionally.

At presentation, her physical exam was benign. Initial cardiac biomarkers (creatine phosphokinase, troponin) and fasting lipid panel were within normal limits. The 12-lead electrocardiogram (ECG) showed normal sinus rhythm without any significant ST-T changes. Chest radiograph showed left perihilar parenchymal scars, surgical clips, and postoperative changes in the chest (Figure 1). Other laboratory studies, including two more sets of cardiac biomarkers, were normal.

She underwent an exercise nuclear stress test which demonstrated indeterminate electrocardiogram (ECG) changes with a small anteroseptal wall defect which appeared to be artifactual. Following the stress test, she developed retrosternal chest pain. Although her ECG did not show any acute changes, repeat serum troponin level was elevated at $0.42 \mathrm{ug} / \mathrm{L}$. Coronary angiography was performed and demonstrated $90 \%$ stenosis of the left main coronary artery ostium, dominant right coronary artery, and no evidence of any atherosclerotic disease or stenosis in other coronary

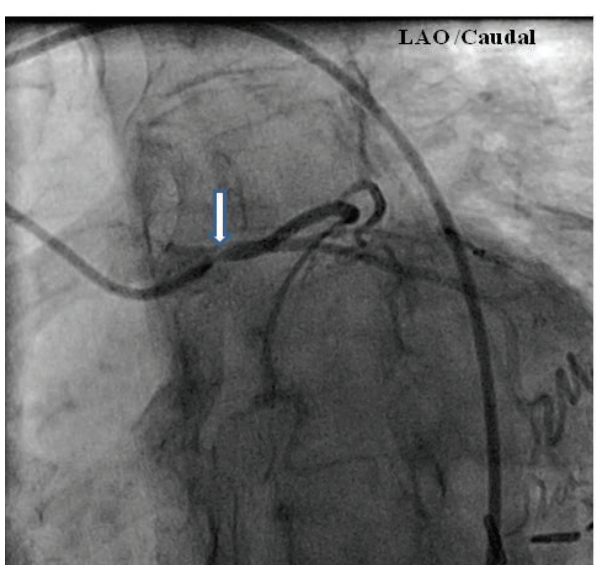

FIGURE 3: Coronary angiography of left main coronary artery in the left anterior oblique (LAO) and caudal view showing severe isolated ostial stenosis (arrow).

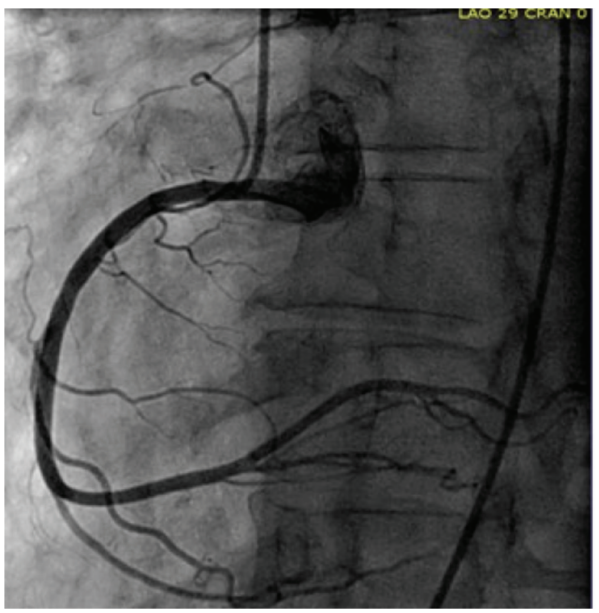

FIGURE 4: Coronary angiography of the right coronary artery in left anterior oblique (LAO) view.

arteries (Figures 2, 3, and 4). Left ventricular ejection fraction was $55 \%$ without any regional wall motion abnormalities.

Syntax score was calculated to be $12 \%$ which predicts comparable outcomes of surgical and angioplastic procedures. Taking into consideration patient's history that may complicate the surgical treatment including her previous surgical interventions, radiation therapy, and chemical therapy, the interventional cardiology team recommended percutaneous coronary intervention (PCI) with stenting of the lesion. Cardiothoracic surgeons were consulted as well for further evaluation and they agreed that surgery will be challenging in this patient, but at the same time they felt that she may tolerate the surgery well, given her functional status, remission from cancer, and the low operative risk (EuroScore is $1.23 \%$ ), and they suggested coronary artery bypass graft (CABG) surgery as a reasonable option due to the high failure rate of stenting ostial lesions and to avoid the possible cardiac arrest associated with the intervention on the stenotic ostium of left main coronary artery.

After discussing both treatment options, the risks and benefits of each procedure with the patient, she decided to 
undergo surgical revascularization. Overnight, she experienced excruciating anginal chest pain without any elevation in cardiac enzymes; as a result she was taken to surgery the next day.

Intraoperatively she was noted to have dense adhesions surrounding the heart. As a result, she developed severe bleeding from the adhesions. Despite aggressive transfusion of red blood cells and platelets, bleeding could not be controlled and she died during surgery. The family refused to allow autopsy to confirm the diagnosis.

\section{Discussion}

This is a case of a 60-year-old female with no classic cardiac risk factors, who developed a stenosis in the ostium of left main coronary artery eight years after undergoing radiation therapy for nonsmall cell lung cancer. She went to the operating room for CABG due to recurrent unstable angina but developed fatal bleeding related to side effects of both radiation and chemotherapy.

Thoracic irradiation can result in injury or inflammation of any of the cardiac structures. CAD has been reported in $5.5-12 \%$ of patients undergoing therapeutic chest radiotherapy, usually manifesting 3-30 years after radiation exposure [3]. Histology of coronary artery tissues obtained from affected patients have demonstrated intimal thickening with minimal extracellular lipid deposits [4]. Although the mechanism of radiation induced CAD (RICAD) is not fully understood, it is believed that radiation may stimulate cytokines and growth factors which in turn induce fibrosis and obstruction [5].

Ostial stenosis is typical of RICAD, since the vessels' openings are usually at the center of the radiation field. One study found that $16 \%$ of isolated coronary ostial stenosis was secondary to radiation therapy [6]. Isolated ostial stenosis of the left main coronary artery following radiation therapy has been reported in eight other cases in the literature [714] (Table 1). These patients were young to middle-aged females (mean age 40 years, range $26-51$ years), with no or few traditional risk factors for CAD. Most presented with typical angina, between 2 and 20 years after radiotherapy. Our patient was older than those previously reported and developed symptoms eight years after therapy. Since RICAD presents about 16 years after radiation exposure and the 5 year survival life in patients with stage IIIA nonsmall cell lung cancer is estimated to be between 9 and 25\%, it is very rare to see RICAD in this group $[1,15]$.

The strategy for revascularization of RICAD is typically the same as for atherosclerotic CAD [1]. Traditionally, CABG has been considered the treatment of choice for left main coronary artery disease (LMCAD) due to its role in increasing the life expectancy in patients with significant stenosis $[16$, 17]. The left internal mammary artery (LIMA) is typically used as a bypass conduit. Despite concerns that radiation might damage the LIMA, increase its fragility, and lead to early graft failure $[18,19]$, recent studies have documented patency rates comparable to that of venous grafts at 5 year follow-up [20, 21], making LIMA grafting a viable option for RICAD as well. Yet cardiac surgery in patients with past chest irradiation can still be technically challenging, with a greater risk of perioperative complications in the presence of pericardial thickening and retrosternal fibrosis. Although an early study has showed good outcomes of CABG treatments in patients with mediastinal radiation therapy, $\mathrm{Wu}$ et al. have found recently that patients with radiation induced heart disease have greater short-term and long-term mortality after cardiovascular surgeries compared to patients in same age and sex who underwent similar surgeries [22, 23]. This study also showed that the standard preoperative risk scores are usually suboptimal in predicting mortality of cardiac surgeries in patients with radiation heart disease [23]. With this in mind, other treatment options such as PCI should always be considered.

There is a growing body of literature supporting PCI with stenting of LMCAD due to atherosclerosis. Some trials have yielded rates of myocardial infarction and mortality similar to CABG, but with better perioperative outcomes [24]. As a result, PCI is now considered an acceptable alternative to surgical revascularization in some patients with LMCAD $[16,25,26]$. This approach could be particularly attractive for patients with radiation-induced adhesions. Ostial stents, though, have been associated with higher rates of restenosis related to their increased risk for proximal or distal displacement [27]. Several new techniques may improve the precision of ostial stent positioning [28], but long-term outcome data are not yet available.

Other comorbidities or medications may also influence the decision to choose percutaneous or surgical revascularization in patients with RICAD. For example, our patient was receiving the gefitinib, a chemotherapeutic agent which decreases platelet function via prostaglandin and thrombaxene and increases the bleeding risk [29]. There are no guidelines or studies on the perioperative management of patients receiving gefitinib. Its half-life is 48 hours, in contrast to 8 hours for the more common antiplatelet agents such as clopidogrel, so prolonged interruption of therapy may be necessary to decrease bleeding risk. We believe that additional preoperative evaluation by a hematologist-oncologist is warranted. Balancing the risk of bleeding against the risk of myocardial infarction or death becomes a complicated and imprecise calculus requiring the input of a multidisciplinary team.

Our case is unique, in that our patient was older at presentation, underwent chest irradiation for lung cancer rather than lymphoma, developed isolated left main coronary ostial stenosis, and her management was complicated by the combined effects of radiation and chemotherapy. More importantly, this case emphasizes the importance of a multidisciplinary team approach and participatory decision making when faced with significant risks of available treatment options.

\section{Disclosure}

Drs. Alsara, Kalavakunta, Alsarah, and Laird-Fick have no relationships to disclose. Dr. Abela is a speaker of Merck, 
TABLE 1: Review of reported cases of isolated ostial stenosis of left main coronary artery following radiation therapy ${ }^{*}$.

\begin{tabular}{lcccccc}
\hline Authors (year) & Gender & Age (Y) & RF & Neoplasm & Latency (Y) & Presenting symptoms \\
\hline Radwaner et al. [7] (1987) & F & 27 & No & Hodgkin's lymphoma & 8 & Angina \\
Grollier et al. [8] (1988) & F & 50 & Smoking & Breast cancer & 5 & Angina \\
Orzan et al. [9] (1995) & F & 26 & No & Hodgkin's lymphoma & 10 & Dyspnea, pleural effusion \\
Takewa et al. [10] (1996) & F & 45 & $/$ & Thymic carcinoid & 3 & Angina \\
Bensaid et al. [11] (1998) & F & 43 & No & Hodgkin's lymphoma & 20 & Myocardial infarction \\
Caus et al. [12] (1999) & F & 47 & Smoking & NH lymphoma & 3 & Angina \\
Victor and Parente [13] (2004) & F & 51 & Dyslipidemia & Hodgkin's lymphoma & 2 & Angina \\
Korosoglou et al. [14] (2012) & F & 34 & No & NH lymphoma & 6 & Angina \\
\hline
\end{tabular}

${ }^{*} \mathrm{~F}$ : female, Y: years, RF: risk factors for coronary artery disease, and NH: non-Hodgkin's.

The case of Takewa et al. [10] is in Japanese. The case of Bensaid et al. [11] is in French.

Abbott, and GlaxoSmithKline. Those relationships are not related to this paper.

\section{Acknowledgment}

Dr. Abela is a recipient of grants from Merck and Novartis.

\section{References}

[1] R. S. Veeragandham and M. D. Goldin, "Surgical management of radiation-induced heart disease," Annals of Thoracic Surgery, vol. 65, no. 4, pp. 1014-1019, 1998.

[2] A. W. Konings, C. T. Smit Sibinga, M. W. Aarnoudse, S. S. de Wit, and H. B. Lamberts, "Initial events in radiation-induced atheromatosis. II. Damage to intimal cells," Strahlentherapie, vol. 154, no. 11, pp. 795-800, 1978.

[3] Z. Miltenyi, K. Keresztes, I. Garai et al., "Radiation-induced coronary artery disease in Hodgkin's disease," Cardiovascular Radiation Medicine, vol. 5, no. 1, pp. 38-43, 2004.

[4] S. I. Dragomanovits, G. L. Lazopoulos, I. N. Tzinieris, and E. N. Deliargyris, "Coronary artery disease following mediastinal irradiation," Hellenic Journal of Cardiology, vol. 48, no. 3, pp. 181-183, 2007.

[5] S. W. Yusuf, S. Sami, and I. N. Daher, "Radiation-induced heart disease: a clinical update," Cardiology Research and Practice, vol. 2011, Article ID 317659, 9 pages, 2011.

[6] R. Pilliere, L. Luquel, D. Brun, F. Jault, I. Gandjbakhch, and J. P. Bourdarias, "Stenosis of the ostium of the left main coronary artery after mediastinal radiotherapy: a case report," Archives des Maladies du Coeur et des Vaisseaux, vol. 84, no. 6, pp. 869872, 1991.

[7] B. A. Radwaner, R. Geringer, A. M. Goldmann, M. J. Schwartz, and H. G. Kemp Jr., "Left main coronary artery stenosis following mediastinal irradiation," American Journal of Medicine, vol. 82, no. 5, pp. 1017-1020, 1987.

[8] G. Grollier, P. Commeau, V. Mercier et al., "Post-radiotherapeutic left main coronary ostial stenosis: clinical and histological study, European Heart Journal, vol. 9, no. 5, pp. 567-570, 1988.

[9] F. Orzan, D. Bellis, F. Mollo, and A. Brusca, "Ostial stenosis of the left main coronary artery in a young woman 10 years after radiation therapy," Cardiovascular Pathology, vol. 4, no. 1, pp. 69-71, 1995.

[10] Y. Takewa, T. Kawata, Y. Yoshida, K. Kawachi, and S. Kitamura, "Radiation-induced coronary ostial stenosis, a case of redo coronary bypass for the restenosis following patch angioplasty,"
Nihon Kyobu Geka Gakkai Zasshi, vol. 44, no. 2, pp. 220-225, 1996.

[11] J. Bensaid, M. Benabbou, C. Goburdhun, C. Medard, A. Guillon, and A. El Kenz, "Ostial stenosis of the common trunk of the left coronary 20 years after mediastinal irradiation," Annales de Cardiologie et d'Angeiologie, vol. 47, no. 10, pp. 732-734, 1998.

[12] T. Caus, I. Canavy, T. Mesana, E. Garcia, and J. Raoul-Monties, "Rescue revascularization for acute coronary occlusion late after radiotherapy," Annals of Thoracic Surgery, vol. 67, no. 1, pp. 236238, 1999.

[13] E. G. Victor and G. B. Parente, "Mediastinal radiation therapy and occlusion of the ostium of the left main coronary artery," Arquivos Brasileiros de Cardiologia, vol. 82, no. 3, pp. 295-300, 2004.

[14] G. Korosoglou, A. V. Kristen, M. Andrassy, H. A. Katus, and S. E. Hardt, "Severe left main coronary stenosis in a young female patient, 6 years after mediastinal radiation therapy for nonHodgkin lymphoma: assessment by coronary angiography and intravascular ultrasound," Clinical Research in Cardiology, vol. 101, no. 4, pp. 317-320, 2012.

[15] C. F. Mountain, "Revisions in the international system for staging lung cancer," Chest, vol. 111, no. 6, pp. 1710-1717, 1997.

[16] R. S. Wright, J. L. Anderson, C. D. Adams et al., "American College of Cardiology Foundation/American Heart Association Task Force on Practice Guidelines. 2011 ACCF/AHA Focused Update Incorporated Into the ACC/AHA 2007 guidelines for the management of patients with unstable angina/Non-STelevation myocardial infarction : a report of the American College of Cardiology Foundation/American Heart Association Task Force on Practice Guidelines," Journal of the American College of Cardiology, vol. 57, no. 19, pp. e215-e367, 2011.

[17] D. P. Taggart, "Coronary artery bypass grafting is still the best treatment for multivessel and left main disease, but patients need to know," Annals of Thoracic Surgery, vol. 82, no. 6, pp. 1966-1975, 2006.

[18] G. L. Hicks Jr., "Coronary artery operation in radiationassociated atherosclerosis: long-term follow-up," Annals of Thoracic Surgery, vol. 53, no. 4, pp. 670-674, 1992.

[19] H. E. Schulman, K. S. Korr, and T. J. Myers, "Left internal thoracic artery graft occlusion following mediastinal radiation therapy," Chest, vol. 105, no. 6, pp. 1881-1882, 1994.

[20] B. Gansera, F. Schmidtler, I. Angelis et al., "Quality of internal thoracic artery grafts after mediastinal irradiation," Annals of Thoracic Surgery, vol. 84, no. 5, pp. 1479-1484, 2007.

[21] M. L. Brown, H. V. Schaff, and T. M. Sundt, "Conduit choice for coronary artery bypass grafting after mediastinal radiation," 
Journal of Thoracic and Cardiovascular Surgery, vol. 136, no. 5, pp. 1167-1171, 2008.

[22] N. Handa, C. G. A. McGregor, G. K. Danielson et al., "Coronary artery bypass grafting in patients with previous mediastinal radiation therapy," Journal of Thoracic and Cardiovascular Surgery, vol. 117, no. 6, pp. 1136-1143, 1999.

[23] W. Wu, A. Masri, Z. B. Popovic et al., "Long-term survival of patients with radiation heart disease undergoing cardiac surgery: a cohort study," Circulation, vol. 127, no. 14, pp. 14761484, 2013.

[24] C. Dubois, J. Dens, P. Sinnaeve et al., "Results of percutaneous coronary intervention of the unprotected left main coronary artery in 143 patients and comparison of 30-day mortality to results of coronary artery bypass grafting," American Journal of Cardiology, vol. 101, no. 1, pp. 75-81, 2008.

[25] S. J. Park, Y. H. Kim, D. W. Park et al., "Randomized trial of stents versus bypass surgery for left main coronary artery disease," New England Journal of Medicine, vol. 364, no. 18, pp. 1718-1727, 2011.

[26] P. E. Buszman, S. R. Kiesz, A. Bochenek et al., "Acute and late outcomes of unprotected left main stenting in comparison with surgical revascularization," Journal of the American College of Cardiology, vol. 51, no. 5, pp. 538-545, 2008.

[27] D. A. Dishmon, A. Elhaddi, K. Packard, V. Gupta, and T. A. Fischell, "High incidence of inaccurate stent placement in the treatment of coronary aorto-ostial disease," Journal of Invasive Cardiology, vol. 23, no. 8, pp. 322-326, 2011.

[28] T. W. Kwan, D. James, Y. Huang, M. Liou, S. Wong, and J. Coppola, "Perfection of precise ostial stent placement," Journal of Invasive Cardiology, vol. 24, no. 7, pp. 354-358, 2012.

[29] S. Kanazawa, K. Yamaguchi, Y. Kinoshita, M. Muramatsu, Y. Komiyama, and S. Nomura, "Gefitinib affects functions of platelets and blood vessels via changes in prostanoids balance," Clinical and Applied Thrombosis/Hemostasis, vol. 11, no. 4, pp. 429-434, 2005. 


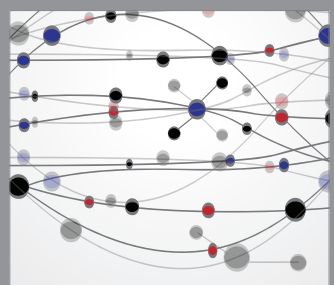

The Scientific World Journal
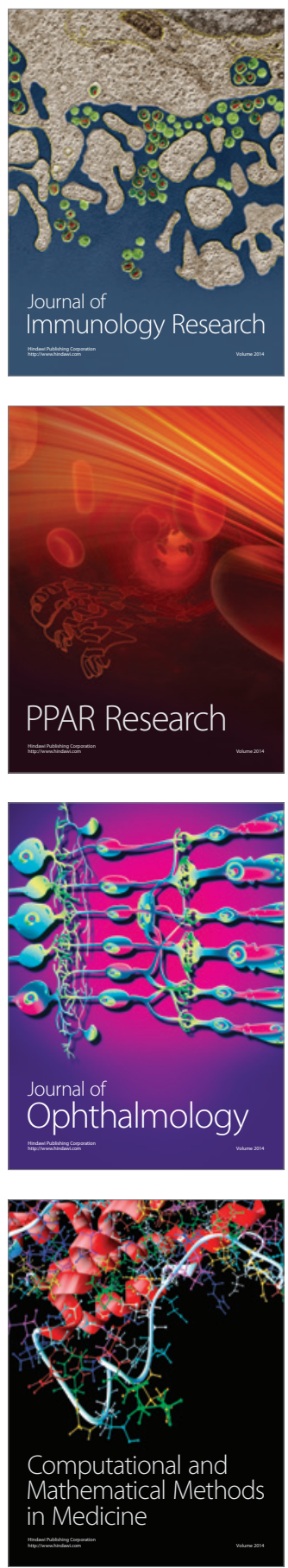

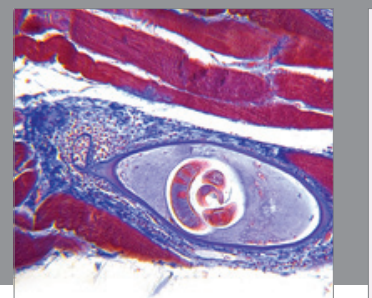

Gastroenterology

Research and Practice
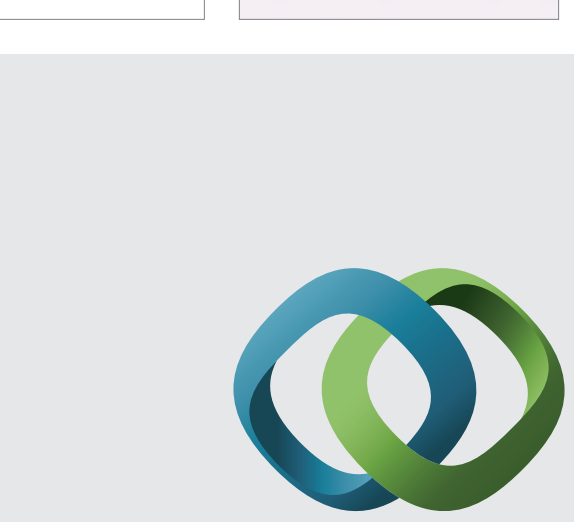

\section{Hindawi}

Submit your manuscripts at

http://www.hindawi.com
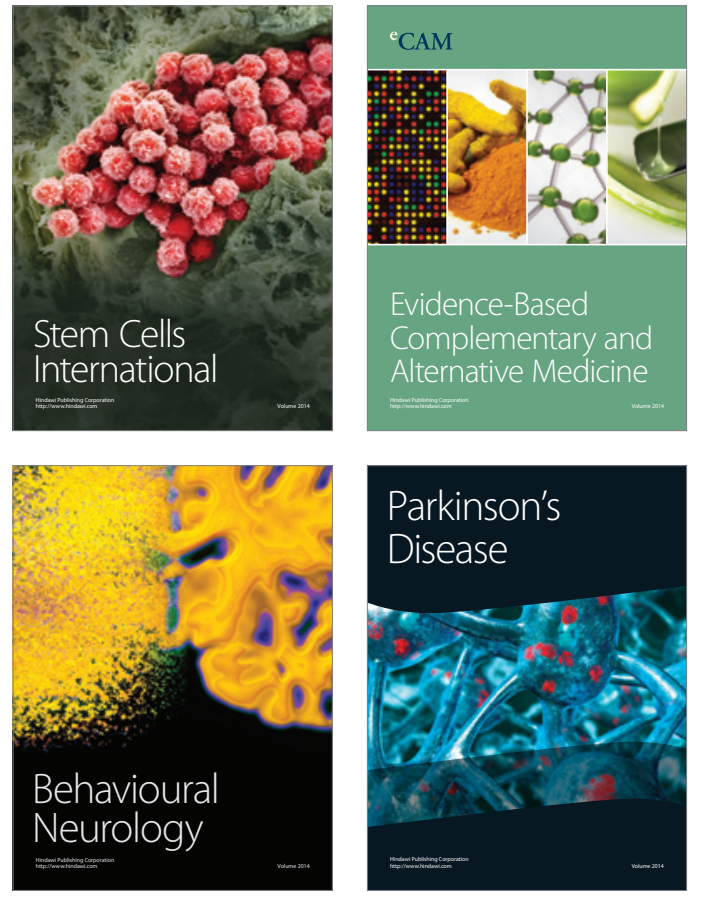
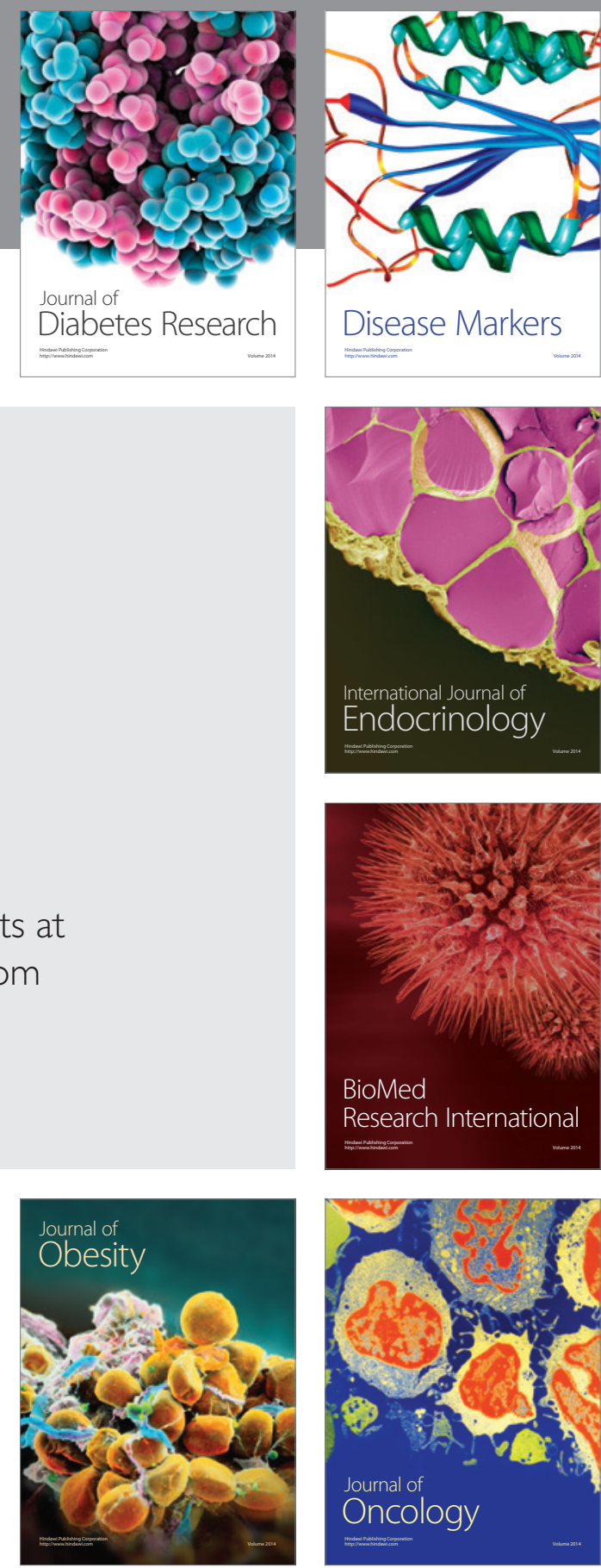

Disease Markers
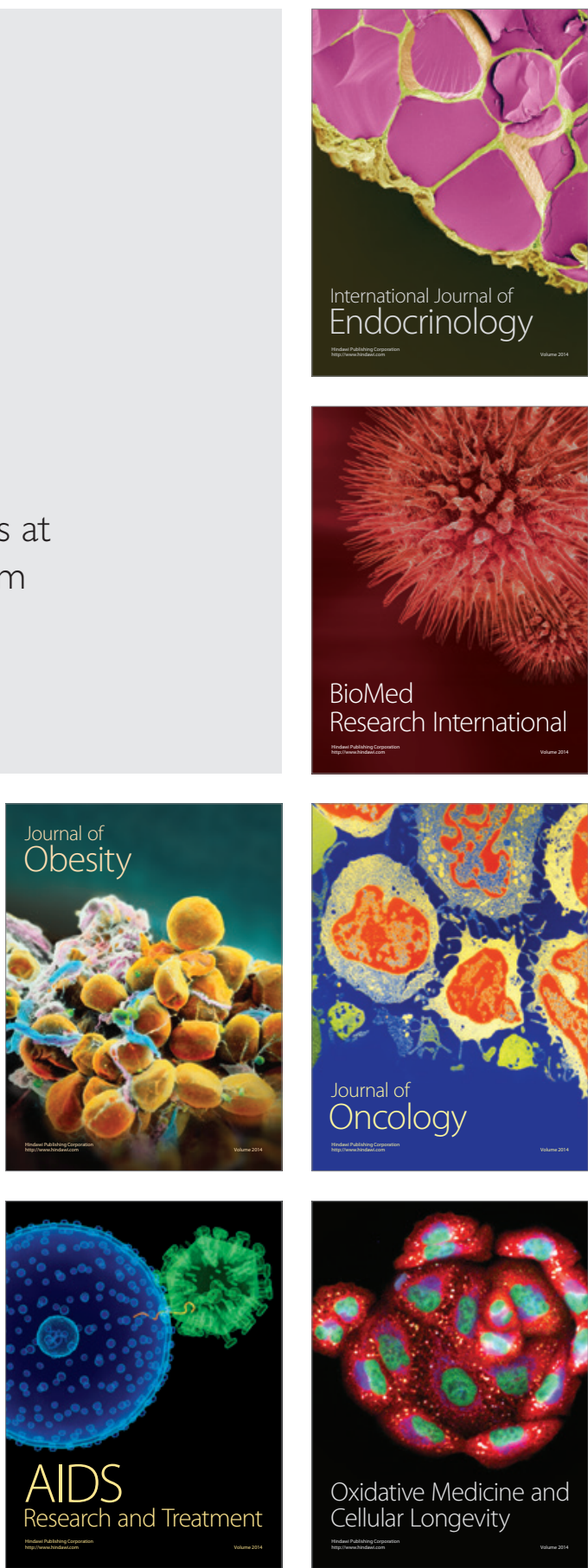